\title{
Infusible Nuclear Fuel Metamaterial for Deep Cosmic-Space Explorations
}

\author{
Boris Udovic, Sezanska 11, 6210 Sezana, SLOVENIA
}

The multistage chain of nuclear-energy conversion-units (thermionics -- magnetohydrodynamic MHD transformers...etc. in avalanche configuration link) gives bigger outcome values for the total efficiency in comparison with a single classic thermodynamic stage alone only. The highest input temperatures are the key for the maximal overall conversion efficiency. Namely, the electric energy availability in huge amounts allows the realization of accelerated plasma ejections in compact and powerful rocket engines via higher temperatures and linear hyper-velocities in comparison with simply expanded hot atomic/molecular gases from thermal nuclear propulsive thrusters - at remarkably lower temperatures. On the other hand the highest obtainable temperatures are hindered in almost all valuable nuclear materials: their reliability is plagued with phase transitions phenomena. The actinide relativistic space/time contractions of the outermost enveloping orbital structure of the itinerant electrons between borderline quantum states are synergistically involved in high temperature dynamics of radionuclide coordination sphere dismantling processes. Inside the often encountered but harshly proven nuclear fuels, which are also stoichiometrically well defined radio-carbide compounds, the thermo-chemical releases of unfastened carbide-ligands relax the inter-bonding angular constrictions and push the overall inner shell distribution to jump into a squeezed sub-coordinated spatial configuration with broken symmetry properties. The spatially/temporally disordered cationic/anionic periodicity of rather isotropically spherical electronic clouds convolves into catastrophic congruent/incongruent phase transitions. The cutting edge breakthrough of the new non-compound chemical synthesis emerges into completely caged but freely rattling radio-nuclides inside of a $3 \mathrm{D}$ spiderlike matrix of tetrahedral amorphous (ta-C) hyperstoichiometric carbon atoms. The rattling-caged isotopes are individually isolated-diluted at some carbon-carbon bondlength units apart from each other. They are efficiently impeded to escape out. The compounded-material fusibility concepts are broken. The metamaterial is far beyond the hyperstoichiometric carbon concept and acquires the characteristics of rather pure tetrahedral amorphous (ta-C) allotropes: the overall working temperature rises up to near $4,000{ }^{\circ} \mathrm{C}$. 